\title{
Chirped-Standing-Wave Acceleration of Ions with Intense Lasers
}

\author{
F. Mackenroth, ${ }^{1, *}$ A. Gonoskov, ${ }^{1,2,3}$ and M. Marklund ${ }^{1}$ \\ ${ }^{1}$ Department of Physics, Chalmers University of Technology, SE-41296 Göteborg, Sweden \\ ${ }^{2}$ Institute of Applied Physics, Russian Academy of Sciences, Nizhny Novgorod 603950, Russia \\ ${ }^{3}$ Lobachevsky State University of Nizhni Novgorod, Nizhny Novgorod 603950, Russia
}

(Received 15 January 2016; published 30 August 2016)

\begin{abstract}
We propose a novel mechanism for ion acceleration based on the guided motion of electrons from a thin layer. The electron motion is locked to the moving nodes of a standing wave formed by a chirped laser pulse reflected from a mirror behind the layer. This provides a stable longitudinal field of charge separation, thus giving rise to chirped-standing-wave acceleration of the residual ions of the layer. We demonstrate, both analytically and numerically, that stable proton beams, with energy spectra peaked around $100 \mathrm{MeV}$, are feasible for pulse energies at the level of $10 \mathrm{~J}$. Moreover, a scaling law for higher laser intensities and layer densities is presented, indicating stable GeV-level energy gains of dense ion bunches, for soon-to-beavailable laser intensities.
\end{abstract}

DOI: 10.1103/PhysRevLett.117.104801

The acceleration of charged particles by intense lasers has become a cornerstone of plasma physics research $[1,2]$, especially aiming at medical applications requiring stable proton beams of 100-200 MeV energy [3], and there are several approaches [4]. In target normal sheath acceleration [5-8], as well as Coulomb explosion of clusters [9] or specially designed targets $[10,11]$, the plasma electrons are heated locally and the remaining ions accelerated by electrostatic fields arising due to the electron cloud's expansion or the ions' repulsion, respectively. The robustness of this process makes it experimentally accessible. But the heating also deprives one of control of the energy transformation, making it difficult to deliver the laser energy to a certain range of ion energies. This inevitably results in a broad ion spectrum, a comparatively low efficiency, and a rather unfavorable scaling law for the ion energy as a function of the laser pulse intensity [1,2]. Collisionless shock acceleration $[12,13]$ can provide monoenergetic ion bunches; however, it typically involves many energy transformation steps also yielding low efficiency and a small number of accelerated ions. Hole boring [14] and light sail (or laser piston) [15-18] imply a more direct and thus controllable energy transfer, yielding higher efficiency as well as more promising spectral properties and scaling laws. However, the balance between the plasma fields and the light pressure introduces an interface susceptible to instabilities [19-22], which strongly limits the acceleration and can even make it experimentally unfeasible.

In this Letter, we propose a new basic approach, which provides an opportunity to overcome the outlined obstacles. The approach relies on placing a thin plasma layer close to a reflecting mirror [see Fig. 1(a)] and locking its electrons between a standing wave's (electric) field antinodes to move them controllably. This leads to the creation of a capacitorlike electric field due to a charge separation between the shifted electrons and the parent ions, the latter being less affected by the ponderomotive force but still being dragged by the electron layer. As the process involves ponderomotive confinement of the electrons from both sides, the locking mechanism does not introduce any interface susceptible to instabilities. The necessary control can be achieved by reflecting a chirped laser pulse from a mirror placed behind the layer. The well-controlled change of the laser's wavelength leads to a changing position of the electric field nodes, similar to studies of ion acceleration in a vacuum [23,24]. Consequently, the motion of the locked electrons can be coordinated with the ion acceleration in the charge separation field, making the ions follow the locked electrons for a long distance. We label this concept chirpedstanding-wave acceleration (CSWA). In principle, it relies only on a tunable laser chirp and a large bandwidth. This is becoming accessible in the ultrahigh-intensity regime, with several joules of laser energy available at a relative

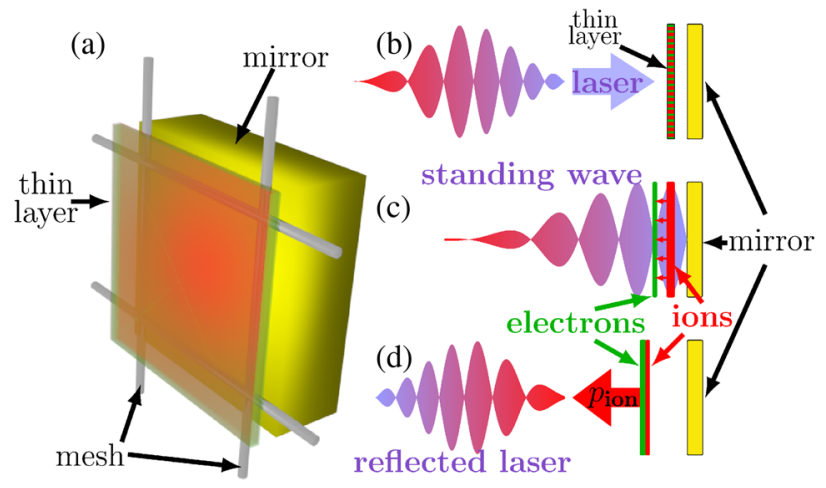

FIG. 1. (a) CSWA target: a high-density mirror and a thin layer fixed in a certain position in front by a micron-thin mesh, leaving voids for the standing wave to form. (b) A chirped laser pulse impinges on the target. (c) A standing wave forms, locks, and displaces the thin layer's electrons, which drag the layer's ions. (d) Ions and electrons propagate away from the mirror. 
bandwidth of $60 \%$ [25] and further development towards higher laser energies at large bandwidths planned [26].

We model the incident laser field as a circularly polarized plane wave of electric field amplitude $E_{0}$ and angular frequency $\omega_{0}$ depending on space-time only via the phase $\eta=t-x / c$, where $c$ is the speed of light and $x(p)$ is the coordinate (momentum) along the laser's propagation direction. Upon reflection from a mirror placed at $x=0$, the laser will form a standing wave $E_{\mathrm{tot}}(t, x)=E(\eta)-E\left(\eta_{+}\right)$, where $\eta_{+}=t+x / c$. The electrons (mass and charge $m_{e}$ and $e$, respectively) of a plasma layer of areal density $\sigma$, placed at the standing wave's first node, will be trapped by the ponderomotive force $F_{\text {pond }}=-e^{2}\left\langle E_{\mathrm{tot}}^{2}(t, x)\right\rangle / 2 m_{e} \omega_{0}^{2}$, where the brackets indicate a temporal average. The ions, on the other hand, are less affected by this force. In contrast, they experience the electron layer's electrostatic force, which varies from $F_{C}=0$ to $F_{C}^{\max }=4 \pi e^{2} \sigma$, depending on the ions' position. To estimate achievable peak ion energies, we use $F_{C}^{\max }$. We determine the layer's areal density $\sigma$ by requiring that, in order to place a layer of particles in front of the mirror and still allow a standing wave to form upon incidence of a laser wave, the thin layer needs to transmit the laser radiation. The electrons will, however, upon incidence of the laser, be accelerated to form a current $j$ emitting radiation that cancels the incoming. Since the current per unit area is limited by $j_{\max }=e \sigma c$, intensities $I>I_{\text {th }}=c /(4 \pi) \times\left(2 \pi j_{\max } / c\right)^{2}=\pi c e^{2} \sigma^{2}$ cannot be canceled and are transmitted through the layer. This is a simple particular case of relativistic self-induced transparency $[27,28]$, as applied in the break-out afterburner [29-33]. In dimensionless units, a radiation field of amplitude $a_{0}=e E_{0} / m_{e} c \omega_{0}$ is transmitted trough a plasma layer with areal density below the threshold $\sigma_{0}=\sigma_{\mathrm{cr}} a_{0} / \pi$, where $\sigma_{\mathrm{cr}}=2 \pi c n_{\mathrm{cr}} / \omega_{0}$ with the critical plasma density $n_{\mathrm{cr}}=m_{e} \omega_{0}^{2} / 4 \pi e^{2}$. The Coulomb attraction to the residual ions of the layer dominates over the light pressure force $F_{l}=(2 / c) I$, until the threshold value $I_{\text {th }}$ is reached. Thus, almost immediately after starting traveling beyond the layer, the electrons get trapped by the newly established standing wave, making this scheme stable against the loss of electrons due to radiation pressure into the mirror. Unlike in the staged ion acceleration scheme [34], the electrons are here locked in the resulting standing wave.

The amplitude $a_{0}$ of the laser is a function of time, and the laser will be transmitted through the thin layer only during the time interval $\tau_{\text {acc }}$ while it is relativistically transparent. We assume the electron layer to be confined by the laser field throughout this whole time interval, whence the ions will be approximately dragged by $F_{C}^{\max }$ for a time $\tau_{\text {acc }}$. The ions' final momentum then is

$$
p_{\text {ion }}=4 \pi e^{2} \sigma \tau_{\text {acc }} .
$$

The ion acceleration thus has two extreme cases where either the charge density vanishes, allowing for an immediate breakthrough of the electric field, or where the layer is so thick that the laser is not able to break through. In both cases, the ions will not be significantly accelerated. Thus, there exists an optimal value for $\tau_{\text {acc. }}$. To estimate this, we neglect the oscillating phase structure of the field and assume that the laser pulse has a Gaussian temporal shape with some bandwidth $\Delta \omega$. The amplitude at the time of breakthrough is then given by $a_{0}\left(\tau_{\text {acc }} / 2\right)=$ $a_{0} \exp \left[-\left(\Delta \omega \tau_{\text {acc }} / 2\right)^{2}\right]$. Inserting the optimal areal density, we find the corresponding areal density

$$
\sigma=\frac{2 c n_{\mathrm{cr}} a_{0}}{\omega_{0}} e^{-\left[\Delta \omega\left(\tau_{\mathrm{acc}} / 2\right)\right]^{2}},
$$

and the final ion momentum is given by

$$
p_{\text {ion }}=2 c m_{e} \omega_{0} a_{0}\left[e^{-\left[\Delta \omega\left(\tau_{\text {acc }} / 2\right)\right]^{2}} \tau_{\text {acc }}\right] .
$$

Only the term in brackets depends on the accelerating time. The maximum of this expression is found at $\tau_{\mathrm{acc}}^{\mathrm{opt}}=\sqrt{2} / \Delta \omega$. We note that the above equation is already optimized for a given set of layer parameters, since the layer's areal charge density is chosen such that relativistic transparency, allowing the pulse to break through the layer, sets in at the time $t=-\tau_{\text {acc }} / 2$. Equation (3) describes a particle of charge $e$ accelerated in a constant field $E_{0} \exp (-1 / 2)$ over a time span $\tau_{\text {acc. }}^{\text {opt }}$ One can thus view the present scheme as a highly efficient field rectifier which turns the laser pulse's transversal into an accelerating longitudinal field of approximately the same amplitude, as also confirmed by numerical simulations [see Fig. 2(e)]. To shift the trapped electron layer controllably, one can use a chirped laser pulse, continuously changing its wavelength and hence also the position of the field nodes. Using a chirp has also been suggested to control other ion acceleration schemes [23,24,35-39]. However, whereas these previous works in the literature build on the model of a linear pulse chirp, we here instead use a model of a laser pulse chirp beyond the linear approximation, in close analogy to the model of a chirped pulse amplifier [40,41]. The model is based on a frequency-dependent phase shift of the field's frequency components (see Supplemental Material [42]). According to this model, a chirped plane wave laser pulse with a Gaussian envelope is given by (see also [41])

$$
\begin{aligned}
& E(\eta)=\frac{E_{0}}{\left(1+\mathcal{C}^{2}\right)^{1 / 4}} e^{-\left[\Delta \omega_{0}(\mathcal{C}) \eta\right]^{2}+i \Sigma(\eta)}, \\
& \Sigma(\eta)=\omega_{0} \eta+\mathcal{C}\left[\left[\Delta \omega_{0}(\mathcal{C}) \eta\right]^{2}+\frac{2 \omega_{0}^{2} \log 2}{\Delta \omega_{0}^{2}}\right]+\frac{\operatorname{atg} \mathcal{C}}{2},
\end{aligned}
$$

with the bandwidth $\Delta \omega_{0}$ (connected to the FWHM pulse duration $\Delta \tau=4 \log 2 \sqrt{1+\mathcal{C}^{2}} / \Delta \omega_{0} \approx 1.2 \mathrm{fs} \times$ $\left.\sqrt{1+\mathcal{C}^{2}} \omega_{0} / \Delta \omega_{0}\right), \mathcal{C}$ a dimensionless parameter quantifying the chirp strength, and $\Delta \omega_{0}(\mathcal{C})=\Delta \omega_{0} /$ $\sqrt{8 \log 2\left(1+\mathcal{C}^{2}\right)}$. The two perpendicular field components of a circularly polarized laser pulse are then $\boldsymbol{E}=[\operatorname{Re}(E(\eta)), \operatorname{Im}(E(\eta))] / \sqrt{2}$. The frequency changes 


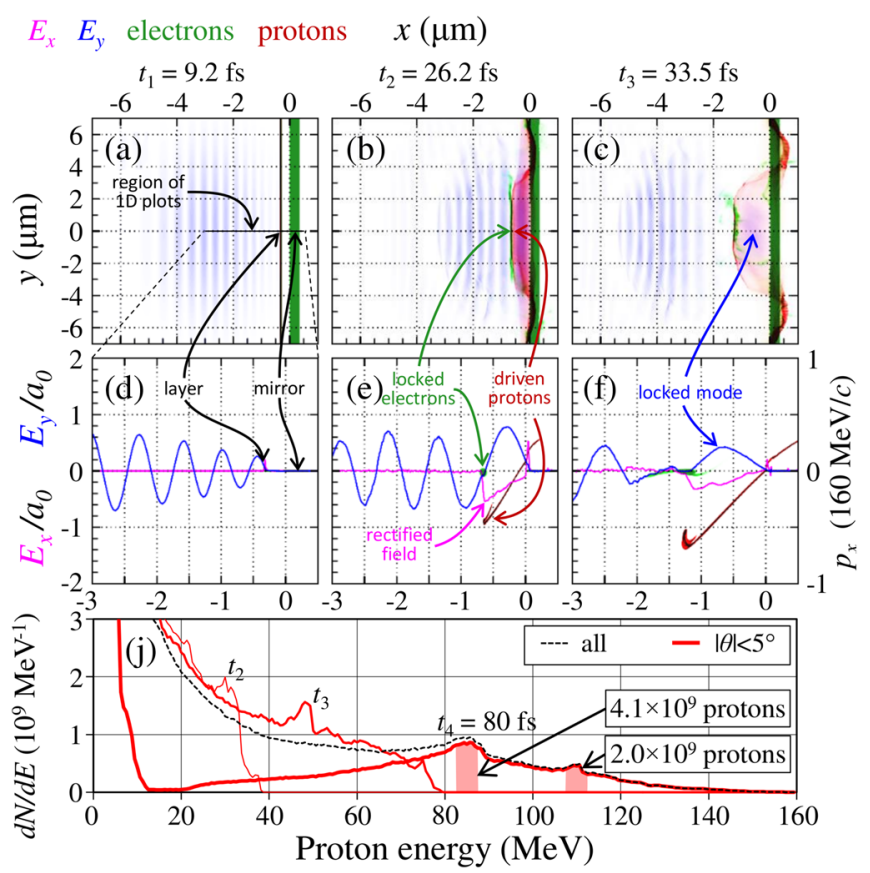

FIG. 2. A 2D PIC simulation for a laser energy $\varepsilon_{0}=30 \mathrm{~J}$, bandwidth $\Delta \omega_{0}=0.5 \omega_{0}$, and chirp $\mathcal{C}=-3.5$. (a)-(c) Transverse field $E_{y}$ (blue), electrons (green), and protons (red) as functions of 2D coordinates. (d)-(f) A $1 \mathrm{D}$ cut additionally showing the longitudinal field component $E_{x}$ (magenta) and particle distributions in phase space $x$ - $p_{x}$. Three time instants are shown: before the layer starts transmitting the incident radiation [(a),(d)], during CSWA with a standing wave formed in the laser's reflection [(b),(e)], or by a mode of radiation locked between the layer and the mirror [(c),(f)]. (j) Spectra of protons from the thin plasma layer (effectively $3 \mathrm{D}$ ) at times $t_{2}, t_{3}$, and a later time $t_{4}$ propagating within a forward cone of $10^{\circ}$ opening angle (red lines) or in an arbitrary direction at $t_{4}$ (black dashed line).

as a function of $\eta$ according to $\omega(\eta)=\omega_{0}+2 \mathcal{C} \Delta \omega_{0}^{2}(\mathcal{C}) \eta$, showing that we go beyond the linear chirp approximation. The standing laser wave formed upon reflecting the field (4) from a mirror fulfills the energy balance $E_{\mathrm{tot}}^{2}(t \rightarrow-\infty, x \rightarrow-\infty)=E_{\mathrm{tot}}^{2}(t \rightarrow+\infty, x \rightarrow-\infty)$, highlighting that no radiation pressure is involved in the acceleration. Neglecting the temporal envelope, the standing wave has its $n$th node in the negative half-space at the purely time-dependent position

$$
x_{\text {node }}(t)=-n \frac{\pi c}{\omega(t)} .
$$

This node will move at a speed

$$
v_{\text {node }}(t)=2 \pi n c \frac{\Delta \omega_{0}^{2}(\mathcal{C})}{\omega^{2}(t)} \mathcal{C},
$$

moving the electrons locked to it. Apparently, at large $n$ the nodes can travel with speeds exceeding $c$. Equating the ponderomotive force to $F_{C}^{\max }$, we find the layer's equilibrium thickness

$$
\Delta x=\frac{\sqrt{2} \pi c \omega_{0}\left(1+\mathcal{C}^{2}\right)^{1 / 4}}{a_{0} \omega^{2}(\eta)} .
$$

Realizing that for a realistic pulse $\omega(\eta) \sim \omega_{0}$ (up to a factor of the order of unity), we see that for a relativistic field strength $\left(a_{0} \gg 1\right)$ the electron layer will be compressed by the ponderomotive force to a thickness $\Delta x \sim \lambda_{0} / a_{0} \ll \lambda_{0}$. Analogously, it can be shown that, for any single particle separated further from the electron layer than twice the derived layer thickness, the laser's ponderomotive force dominates over the Coulomb attraction between the electron and ion layers, ensuring the stability of the suggested scheme throughout the whole duration of the laser pulse. Inserting the field model from Eq. (4) into the optimized Eq. (3), we obtain

$$
p_{\mathrm{ion}}^{\mathrm{opt}} \approx 4 m_{e} c \frac{\omega_{0}}{\Delta \omega_{0}} a_{0}\left(1+\mathcal{C}^{2}\right)^{1 / 4} .
$$

The ions require a finite momentum even for $\mathcal{C} \equiv 0$ as an artifact of modeling the accelerating field as constant over $\tau_{\text {acc }}$ in Eq. (1). We thus apply Eq. (8) only for $|\mathcal{C}|>1$. According to Eq. (8), the ions' final momentum scales as $p_{\text {ion }}^{\text {opt }} \sim\left(1+\mathcal{C}^{2}\right)^{1 / 4}$, while the charge surface density scales as $\sigma \sim\left(1+\mathcal{C}^{2}\right)^{-1 / 4}$. The areal charge current density $j=\sigma p_{\mathrm{ion}}^{\mathrm{opt}} / m \sim a_{0}^{2}$, however, is independent of the chirp. We conclude that, provided one maintains the optimum surface density condition, the pulse chirp is a tunable parameter to trade maximum particle energies for the number of accelerated particles. Furthermore, it even allows us to tune between high ion energies and a monoenergetic spectrum, since for large chirps some of the ions outrun the locked electrons and are no longer accelerated. They start circulating around the locked electrons [see Figs. 2(e) and 2(f)], compressing the protons' spectrum. For nonrelativistic ions of mass $m_{\text {ion }}$, Eq. (8) corresponds to a final ion velocity $v_{\text {ion }} \approx p_{\text {ion }}^{\text {opt }} / m_{\text {ion }}$. Since the ions need to be close to, but cannot overtake, the electron layer locked to the field nodes, we expect the optimal ion acceleration if $v_{\text {ion }} \approx v_{\text {node }}(1.5 \Delta \tau)$, where $1.5 \Delta \tau$ is a suitable time scale for the ions to approach their final velocity. For $|\mathcal{C}| \gg 1$, we then find the following chirp value for the optimal ion acceleration:

$$
\mathcal{C}^{\mathrm{opt}} \approx-\left[\frac{m_{\mathrm{ion}}}{m_{e}} \frac{\pi}{16 \log 2} \frac{\left(\frac{\Delta \omega_{0}}{\omega_{0}}\right)^{3}}{a_{0}\left[1-1.5\left(\frac{\Delta \omega_{0}}{\omega_{0}}\right)\right]^{2}}\right]^{2 / 3} .
$$

The scaling $\mathcal{C}^{\mathrm{opt}} \sim a_{0}^{-2 / 3}$ is due to the fact that at higher pulse energies higher final ion velocities require faster node propagation.

The dominant higher-dimensional effect is dephasing: In the one-dimensional analysis, one neglects the accelerating field's dependence on the distance between the proton and electron layers, effectively ending the acceleration once the layers are separated further than their transverse size. Since the layers' transverse size is, however, of the order of the laser spot, i.e., several microns and the longitudinal motion is subwavelength, one expects dephasing to be negligible. 
Quantitatively, inserting the optimal areal charge density from Eq. (2) into the accelerating force $F_{C}^{\max }$, we estimate the position of the accelerated ion layer $x_{\text {ion }}(t)=F_{C} /$ $2 m_{\text {ion }} t^{2}+x_{\text {ion }}(0)$. Equating the resulting distance between the ion layer and the electrons, locked to the field nodes at $x_{\text {node }}(t)$, to the transverse spot size leads to a cubic equation in time, which we have investigated numerically (see Supplemental Material [42]). It showed that for all parameters studied in this work the time scale for dephasing is significantly longer than the pulse duration $\Delta \tau$, when the electrons are no longer driven by the standing wave and dephasing can no longer occur. We thus employ the presented one-dimensional analysis as a qualitative model of CSWA.

To test the analytical model, we performed numerical experiments. We note that due to the laser's circular polarization and circular focal spot there is no preferred transverse direction. Thus, the formation of instabilities and the beam's divergence can be studied already in a 2D simulation. Also, the total particle density in a 3D geometry can be deduced from a 2D simulation resolving only the laser's propagation direction $x$ and one perpendicular coordinate $y$ via $d N=2 \pi r(d N / d z) d z$, where $d N / d z$ is the particle density in the unresolved third coordinate and $r$ the distance from the laser axis in the $y$ direction. We performed a 2D particle-in-cell (PIC) simulation, using the code PICADOR [43], to demonstrate CSWA to efficiently produce an ion beam with low divergence which is stable against plasma instabilities (see Supplemental video [42] and Fig. 2). We used Eq. (4) to model a circularly polarized laser pulse with central wavelength $\lambda_{0}=810 \mathrm{~nm}$ under normal incidence, focused to a circular spot of $d_{0}=7.5 \mu \mathrm{m}$ diameter onto a thin layer of electrons and protons (mass $m_{p}$ and charge $-e$ ) in front of a mirror consisting of electrons and heavy ions (mass $20 m_{p}$ and charge $-e$ ) in a simulation box with $2048 \times 3072$ cells and a size of approximately $10 \times 20 \mu \mathrm{m}$. The mirror is placed at $x=0$ and the thin plasma layer at $x=0.4 \lambda_{0}$, to account for imperfect placement of the layer not exactly at $\lambda_{0} / 2$. We checked that with a tolerance of $\pm \lambda_{0} / 4$ changes in the layer's initial position, possibly due to fabrication inaccuracy, do not significantly affect CSWA, since the standing wave's nodes capture all electrons within half a wavelength, establishing an efficient autostabilization of CSWA. We choose the initial conditions such that at the simulation start the center of the chirped laser pulse is placed approximately 5 times its spatial width in front of the layer. First, the incident laser radiation is reflected by the layer, causing a minor internal shift of the layer's electrons and a subsequent proton acceleration in the positive $x$ direction. Surpassing the threshold intensity $(t \approx 12 \mathrm{fs}$ ), the incident radiation starts to penetrate the layer, forms a standing wave in front of the mirror, and captures the electrons within less than 3 fs. They follow the field node, and the generated quasistatic longitudinal field starts to accelerate protons in the negative $x$ direction $(t \approx 15-30 \mathrm{fs})$ with the electron and proton layers significantly compressed and stabilized by the laser's ponderomotive force [see
Fig. 2(b)]. Note that the longitudinal sheath field, formed on the mirror surface through electron expulsion by the laser, points in the positive $x$ direction, due to the quasi-onedimensional geometry [see Fig. 2(e)], not disturbing the proton acceleration. The acceleration continues after the laser radiation has decoupled from the electron layer within this second stage, the standing wave being provided by a radiation mode locked between the layer and the mirror $(t \approx 30-80 \mathrm{fs}$ ), accelerating the protons to energies beyond $140 \mathrm{MeV}$. The protons' distribution is clearly peaked at high energies and in position, proving the strong suppression of plasma instabilities in CSWA even at late times [see Figs. 2(c), 2(f), and 2(j)]. All high-energy protons propagate within a narrow cone around the negative $x$ axis, indicating a low beam divergence. Energy selecting protons, as is customarily done [44,45], yields $4 \times 10^{9}\left(2 \times 10^{9}\right)$ protons in a $\pm 2.5 \mathrm{MeV}$ window around $85 \mathrm{MeV}(110 \mathrm{MeV})$ without any further optimization [see Fig. 2(j)]. As indicated above, however, an increased monochromaticity is also intrinsically achievable at the cost of reduced ion energies.

We confirmed that, for laser spot sizes $d_{0} \gtrsim 7 \mu \mathrm{m}$, the protons' spectral properties from a $2 \mathrm{D}$ simulation were in good agreement with those from a 1D simulation. This further indicates that CSWA is well described by a 1D approximation. For smaller spot sizes in 2D simulations, the protons' monochromaticity is reduced while their maximal energies are still in good agreement with those from 1D simulations. Then, to highlight CSWA's wide applicability range and tunability through the chirp, we performed a parameter scan of the maximal proton energies with 1D simulations. We assumed a reduced spot size of $d_{0}=5 \mu \mathrm{m}$ and the plasma layer to be initially placed at $x=\lambda_{0} / 2$, with all other parameters unchanged compared to the previous example. We then varied the total pulse energy $\varepsilon_{0}$ and the chirp $\mathcal{C}$ (see Fig. 3). Comparing the results to $\mathcal{C}^{\text {opt }}$ from Eq. (9), for small pulse energies we find very good agreement with the theory. For large pulse energies, on the other hand, the chirp of the optimal proton acceleration becomes larger than $\mathcal{C}^{\text {opt }}$, probably due to $\mathcal{C}^{\text {opt }}$ reducing to small values and simultaneously the second stage of the acceleration becoming dominant.

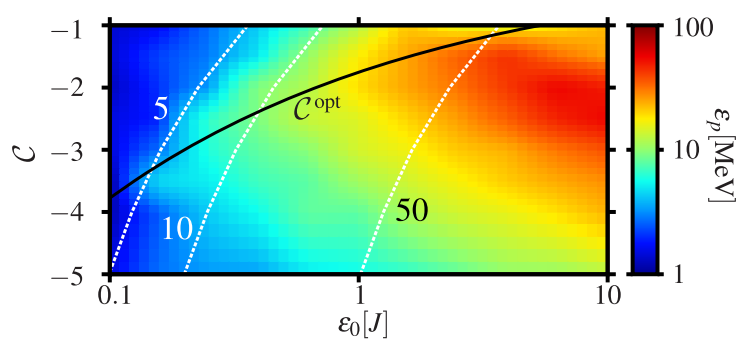

FIG. 3. Maximum proton energy $\varepsilon_{p}$ (energy above which $1 \%$ of all protons lie) as a function of the pulse chirp $\mathcal{C}$ and the laser energy $\varepsilon_{0}$. All parameters are as in Fig. 2 except $\Delta \omega_{0}=0.3 \omega_{0}$. Black line: Optimum chirp from Eq. (9). White dashed lines and adjacent numbers: Maximum proton energy from Eq. (8) in MeV. 
The displayed proton energy thresholds (dashed lines), obtained from Eq. (8), are also well reproduced for small chirp values, while for $\mathcal{C}>\mathcal{C}^{\text {opt }}$ the acceleration's efficiency reduces, since the protons catch up with the electron layer, preventing them from achieving high energies. Thus, CSWA is demonstrated to yield proton energies of the order of $\varepsilon_{p} \approx p_{p}^{2} / 2 m_{p} \sim 100 \mathrm{MeV}$ for pulse energies $\varepsilon_{0} \sim 10 \mathrm{~J}$ while simultaneously offering control over their phase space distribution. Consequently, Eq. (8) indicates that, at $\varepsilon_{0} \sim 100 \mathrm{~J}$, CSWA could admit controlling dense and collimated proton beams of up to $\varepsilon_{p} \sim 1 \mathrm{GeV}$.

We presented a novel approach to laser ion acceleration, efficiently converting a laser's transverse into an accelerating field and insusceptible to the formation of plasma instabilities. We demonstrated its feasibility over a wide range of realistic parameters and presented a scaling law indicating the feasibility of $\mathrm{GeV}$-level ion acceleration. We highlighted a unique tunability, enabling a trade-off between the number and energies of accelerated particles.

The authors acknowledge valuable discussions with C.-G. Wahlström, O. Lundh, and J. Magnusson and technical support by the PICADOR development team (especially by S. Bastrakov) as well as financial support by the Wallenberg Foundation within the grant "Plasma based compact ion sources" (PLIONA), and by the Russian Science Foundation Project No. 16-12-10486 (A. G.). The simulations were performed on resources provided by the Swedish National Infrastructure for Computing (SNIC).

*felix.mackenroth@chalmers.se

[1] H. Daido, M. Nishiuchi, and A. S. Pirozhkov, Rep. Prog. Phys. 75, 056401 (2012).

[2] A. Macchi, M. Borghesi, and M. Passoni, Rev. Mod. Phys. 85, 751 (2013).

[3] S. S. Bulanov et al., Med. Phys. 35, 1770 (2008).

[4] F. Mackenroth, A. Gonoskov, and M. Marklund (to be published).

[5] M. Roth et al., Phys. Rev. ST Accel. Beams 5, 061301 (2002).

[6] P. Mora, Phys. Rev. Lett. 90, 185002 (2003).

[7] T. E. Cowan et al., Phys. Rev. Lett. 92, 204801 (2004).

[8] M. Passoni, L. Bertagna, and A. Zani, New J. Phys. 12, 045012 (2010).

[9] T. Ditmire, J. W. G. Tisch, E. Springate, M. B. Mason, N. Hay, R. A. Smith, J. Marangos, and M. H. R. Hutchinson, Nature (London) 386, 54 (1997).

[10] T. Z. Esirkepov et al., Phys. Rev. Lett. 89, 175003 (2002).

[11] S. S. Bulanov et al., Phys. Rev. E 78, 026412 (2008).

[12] L. O. Silva, M. Marti, J. R. Davies, R. A. Fonseca, C. Ren, F. Tsung, and W. B. Mori, Phys. Rev. Lett. 92, 015002 (2004).

[13] D. Haberberger, S. Tochitsky, F. Fiuza, C. Gong, R. A. Fonseca, L. O. Silva, W. B. Mori, and C. Joshi, Nat. Phys. 8, 95 (2012).

[14] T. Schlegel, N. Naumova, V. T. Tikhonchuk, C. Labaune, I. V. Sokolov, and G. Mourou, Phys. Plasmas 16, 083103 (2009).
[15] T. Esirkepov, M. Borghesi, S. V. Bulanov, G. Mourou, and T. Tajima, Phys. Rev. Lett. 92, 175003 (2004).

[16] S. V. Bulanov, E. Y. Echkina, T.Z. Esirkepov, I. N. Inovenkov, M. Kando, F. Pegoraro, and G. Korn, Phys. Rev. Lett. 104, 135003 (2010).

[17] A. Henig et al., Phys. Rev. Lett. 103, 245003 (2009).

[18] S. Kar et al., Phys. Rev. Lett. 109, 185006 (2012).

[19] F. Pegoraro and S. V. Bulanov, Phys. Rev. Lett. 99, 065002 (2007).

[20] V. Khudik, S. A. Yi, C. Siemon, and G. Shvets, Phys. Plasmas 21, 013110 (2014).

[21] A. Sgattoni, S. Sinigardi, L. Fedeli, F. Pegoraro, and A. Macchi, Phys. Rev. E 91, 013106 (2015).

[22] C. A. J. Palmer et al., Phys. Rev. Lett. 108, 225002 (2012).

[23] F. Peano, J. Vieira, R. A. Fonseca, R. Mulas, G. Coppa, and L. O. Silva, IEEE Trans. Plasma Sci. 36, 1857 (2008).

[24] F. Peano, J. Vieira, L. O. Silva, R. Mulas, and G. Coppa, New J. Phys. 10, 033028 (2008).

[25] Z. Major et al., Review of Laser Engineering 37, 431 (2009).

[26] M. Zepf, in Proceedings of the ELI-Beamlines Inaugural Meeting, 2015 (unpublished); M. Zepf (private communication).

[27] V. A. Vshivkov, N. M. Naumova, F. Pegoraro, and S. V. Bulanov, Phys. Plasmas 5, 2727 (1998).

[28] A. Macchi, S. Veghini, and F. Pegoraro, Phys. Rev. Lett. 103, 085003 (2009).

[29] L. Yin, B. J. Albright, B. M. Hegelich, and J. C. Fernández, Laser Part. Beams 24, 291 (2006).

[30] B. M. Hegelich, B. J. Albright, J. Cobble, K. Flippo, S. Letzring, M. Paffett, H. Ruhl, J. Schreiber, R. K. Schulze, and J. C. Fernández, Nature (London) 439, 441 (2006).

[31] D. Jung et al., New J. Phys. 15, 023007 (2013).

[32] D. Jung et al., Phys. Plasmas 20, 083103 (2013).

[33] B. M. Hegelich et al., New J. Phys. 15, 085015 (2013).

[34] A. A. Gonoskov, A. V. Korzhimanov, V. I. Eremin, A. V. Kim, and A. M. Sergeev, Phys. Rev. Lett. 102, 184801 (2009).

[35] F. Peano, J. Vieira, R. Mulas, G. Coppa, R. Bingham, and L. O. Silva, Plasma Phys. Controlled Fusion 51, 024006 (2009).

[36] B. J. Galow, Y. I. Salamin, T. V. Liseykina, Z. Harman, and C. H. Keitel, Phys. Rev. Lett. 107, 185002 (2011).

[37] A. Popp et al., Phys. Rev. Lett. 105, 215001 (2010).

[38] V. B. Pathak, J. Vieira, R. A. Fonseca, and L. O. Silva, New J. Phys. 14, 023057 (2012).

[39] H. Vosoughian, Z. Riazi, H. Afarideh, and E. Yazdani, Phys. Plasmas 22, 073110 (2015).

[40] G. A. Mourou, T. Tajima, and S. V. Bulanov, Rev. Mod. Phys. 78, 309 (2006).

[41] B. E. A. Saleh and M. C. Teich, Fundamentals of Photonics, 2nd ed. (Wiley, Hoboken, 2013).

[42] See Supplemental Material at http://link.aps.org/ supplemental/10.1103/PhysRevLett.117.104801 for a video of CSWA, a comprehensive derivation of the chirped pulse model and a numerical analysis of the dephasing length.

[43] S. Bastrakov, R. Donchenko, A. Gonoskov, E. Efimenko, A. Malyshev, I. Meyerov, and I. Surmin, J. Comput. Sci. 3, 474 (2012).

[44] S. Busold et al., Nucl. Instrum. Methods Phys. Res., Sect. A 740, 94 (2014).

[45] S. Agosteo et al., Nucl. Instrum. Methods Phys. Res., Sect. B 331, 15 (2014). 